\title{
Bilinear Modelling and Bilinear PI Control of Directional Drilling
}

\author{
Isonguyo J. Inyang \\ School of Aerospace, \\ Transport and Manufacturing, \\ Cranfield University, \\ Bedfordshire, MK43 0AL, UK. \\ Email: i.inyang@cranfield.ac.uk
}

\author{
James F. Whidborne \\ School of Aerospace, \\ Transport and Manufacturing, \\ Cranfield University, \\ Bedfordshire, MK43 0AL, UK. \\ Email: j.f.whidborne@ cranfield.ac.uk
}

\author{
Martin T. Bayliss \\ Schlumberger Oilfield UK Plc. \\ Stonehouse Technology Centre, Brunel Way, \\ Stroudwater Business Park, Stonehouse, \\ Gloucestershire, GL10 3SX, UK. \\ Email: mbayliss@slb.com
}

\begin{abstract}
This paper presents the design of an inclinationand azimuth-hold controllers and their subsequent stability and performance analysis for directional drilling tools as typically used in the oil industry. Using an input transformation developed in earlier work that partially linearizes and decouples the plant dynamics of the directional drilling tool, a bilinear model of the directional drilling tool is developed and is used as the basis for Bilinear PI controller design. Results for a transient simulation of the proposed BPI controller are presented and compared with that of the PI controller of the earlier work. It is presented that BPI controller gives more consistent responses over a broader operating range compared to the PI controller. In addition, the effect of time delay on the feedback measurements with respect to the stability and performance is investigated in the simulations.
\end{abstract}

\section{INTRODUCTION}

In recent years the oil and gas industry has sought to extend the life of existing wells and to exploit smaller and hitherto difficult-to-commercialize reservoirs by using directional drilling and rotary steerable drilling tools [1]. Steerable tools enable the direction of the well propagation to be directed as required either by passive steering control from the surface using fixed-bend positive displacement motors [2], [3] or by steering the wellbore propagation downhole using a rotary steerable system (RSS) drilling tool. Directional drilling, by either approach, is essentially attitude control, that is, concerned with controlling azimuth and inclination [4].

Figure 1 shows the main components of a typical RSS directional drilling system as discussed in this paper. In Figure 1 , it can be seen that the combination of the drill string and BHA (Bottom Hole Assembly) can be viewed as a long flexible prop-shaft transmitting torque to the bit downhole. In the annular clearance between the well bore and the drill string/BHA mud returns to the surface as a means of lubrication and cuttings transport (amongst other functions) after being pumped from the surface through the center of the drill string/BHA. The drill string/BHA is suspended from the block set weight on bit actuator at the drill rig on the surface, which is also where the top drive rotational actuator is situated.

This paper particularly addresses a generic (toolindependent) attitude control algorithm developed for use with directional drilling tools. The importance of attitude control is highlighted by [4] and [2] who propose control

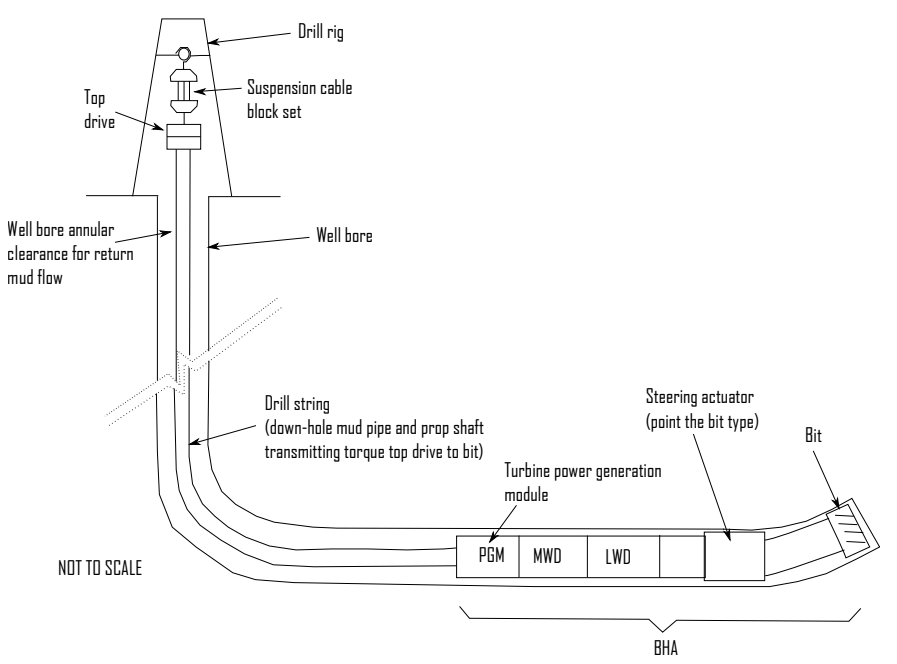

Fig. 1. Schematic of main RSS directional drilling system components

strategies for the direction of wellbore propagation based on holding the tool-face angle. Reference [4] discussed the need for closed-loop 'shoot and forget systems' and state that the major challenge has been azimuth control. Many of the attitude control strategies presented in the literature are discussed in the context of specific tool architectures; [2], [4] and [5] are typical examples. Another interesting example is [3] which describes a system for controlling attitude using two-way telemetry communication links with the surface; the steering correction, evaluated from the telemetry, is computed and then manually downlinked to the tool.

In the modelling of physical systems, the dynamics are often approximated as linear models that are obtained by a first order Taylor series approximation of the non-linear model at a particular point of operation. It is clear that such linear models may be inaccurate over a wider range of operation, hence bilinear models have been proposed to more accurately describe the nonlinear systems (see, for example, [6], [7]). Bilinear models can characterize nonlinear properties more correctly than linear models, and hence broaden the range of adequate performance. In this paper, a bilinear model of the directional drilling tool is developed by applying a

Published by IEEE. This is the Author Accepted Manuscript.

This article may be used for personal use only. The final published version is available online at 10.1109/ CONTROL.2016.7737607. Please refer to any applicable publisher terms of use. 
Carleman Bilinearization technique, described in [8], on a partially linearized and decoupled plant dynamics of the directional drilling tool developed in the earlier work of [9]. The generalized state space representation of a multiple-input multiple-output (MIMO) bilinear system is expressed as [10]:

$$
\begin{aligned}
& \dot{x}=A x+\left(B+\sum_{i=1}^{N} x_{i} M_{i}\right) u \\
& y=C x
\end{aligned}
$$

where $A, B, C$ and $M_{i}$ are constant matrices of suitable dimensions, $y \in \mathbb{R}^{q \times 1}$ represents the output to be controlled, $u$ $\in \mathbb{R}^{m \times 1}$ denotes the control vector, and $x \in \mathbb{R}^{n \times 1}$ represents the vector of state variables.

Furthermore, and extending the previous work of [9], this paper describes an approach for designing a Bilinear Proportional plus Integral (BPI) controller that has the control objective, once engaged, of automatically holding the inclination and azimuth of the tool at nominally constant values as the well is propagated. Usually the tool is manually controlled to the desired location and orientation, the attitude (azimuth and inclination) is measured, and then these values are subsequently used as the demand attitude for the control algorithm. Unstructured and structured uncertainty analysis of the robust stability and performance of pole placement, $H_{\infty}$ and Linear Quadratic Gaussian (LQG) controllers were performed [9], [11], [12]. Here, the BPI controller, which is applied for the attitude control of the directional drilling tool, and illustrated in this paper, is cogitated to present improved performance over a broader operating limit.

This paper is structured so that the design method for the BPI control design is presented, starting with a statement of the plant model; plant bilinearization; control design; and finally, incorporating it into a transient simulation using the plant model in nonlinear form together with the necessary control architecture. The control design and analysis was performed using MATLAB and its associated Control System toolbox commands and the subsequent transient simulations were performed in Simulink.

\section{SUMMARY OF EARLIER WORK}

\section{A. Tool Kinematics}

The plant model is derived from kinematic considerations as detailed in [9]. The resulting governing equations can be stated as:

$$
\begin{gathered}
\dot{\theta}_{\text {inc }}=V_{\text {rop }}\left(U_{\mathrm{dls}} \cos U_{\mathrm{tf}}-V_{\mathrm{dr}}\right) \\
\dot{\theta}_{\mathrm{azi}}=\frac{V_{\mathrm{rop}}}{\sin \theta_{\mathrm{inc}}}\left(U_{\mathrm{dls}} \sin U_{\mathrm{tf}}-V_{\mathrm{tr}}\right)
\end{gathered}
$$

where $\theta_{\text {inc }}$ is the inclination angle, $\theta_{\text {azi }}$ is the azimuth angle, $U_{\mathrm{tf}}$ is the tool-face angle control input, $U_{\mathrm{dls}}$ is the curvature $\left(K_{\mathrm{dls}} \times\right.$ duty cycle), $K_{\mathrm{dls}}$ is the open loop curvature capability of the tool, $V_{\mathrm{dr}}$ is the drop rate disturbance $\left(V_{\mathrm{dr}}=\alpha \sin \theta_{\mathrm{inc}}\right), V_{\mathrm{tr}}$ is the turn rate bias disturbance and $V_{\text {rop }}$ is the rate of penetration and is an uncontrolled parameter.
Note, the subscript 'dls' has been used to reflect that in the oil industry curvature is often referred to as 'dogleg severity'. As detailed in [9], engineering constraints include the control inputs $U_{\mathrm{dls}}$ and $U_{\mathrm{tf}}$ being discretized into duty cycles known as 'drilling cycles' and that the tool-face input $U_{\mathrm{tf}}$ is subject to first order lag dynamics. Additionally, the on-tool feedback measurements of $\theta_{\text {inc }}$ and $\theta_{\text {azi }}$ are subject to pure delays dependent on $V_{\text {rop }}$ as a consequence of the relevant sensors being spatially offset from the drill bit (the inertial datum). These controller and sensor dynamics are ignored for the controller design.

\section{B. Partially Linearized and Decoupled Plant}

The MIMO open loop plant can be partially linearized and decoupled using the following transformation:

$$
\begin{gathered}
U_{\mathrm{tf}}=\operatorname{ATAN2}\left(U_{\mathrm{azi}}, U_{\mathrm{inc}}\right) \\
U_{\mathrm{dls}}=K_{\mathrm{dls}} \sqrt{\left(U_{\mathrm{azi}}\right)^{2}+\left(U_{\mathrm{inc}}\right)^{2}}
\end{gathered}
$$

where $U_{\text {azi }}$ and $U_{\text {inc }}$ are virtual control of azimuth and inclination, respectively. Substituting (5) and (6) into (3) and (4) with the drop and turn disturbances removed, gives the open loop dynamics as:

$$
\begin{gathered}
\dot{\theta}_{\mathrm{inc}}=V_{\mathrm{rop}} K_{\mathrm{dls}} U_{\mathrm{inc}} \\
\dot{\theta}_{\mathrm{azi}}=\frac{V_{\mathrm{rop}}}{\sin \theta_{\mathrm{inc}}} K_{\mathrm{dls}} U_{\mathrm{azi}}
\end{gathered}
$$

Therefore the control transformations, (5) and (6), partially linearize and decouple the governing equations.

\section{Plant Bilinearization}

In this section, a technique called Carleman Bilinearization described in [8], is applied on the partially linearized and decoupled plant, (7) and (8), to obtain a bilinear model of the directional drilling tool. Ignoring disturbances, (7) and (8) are re-written as

$$
\begin{aligned}
& \dot{\theta}_{\mathrm{inc}}=a U_{\mathrm{inc}} \\
& \dot{\theta}_{\mathrm{azi}}=\frac{a}{\sin \theta_{\mathrm{inc}}} U_{\mathrm{azi}}
\end{aligned}
$$

where $a=V_{\text {rop }} K_{\mathrm{dls}}$. Defining an augmented state vector for the Carleman Bilinearization as:

$$
x^{\otimes}=\left[x_{1}, x_{1}^{(2)}, x_{1}^{(3)}, x_{1}^{(4)}, \ldots, x_{1}^{(N)}, x_{2}\right]^{T}
$$

where $x_{1}=\theta_{\text {inc }}, x_{2}=\theta_{\text {azi }}$ and $x_{1}^{(i)}=\frac{d}{d t}\left[\left(x_{1}\right)^{i}\right]=i \dot{x}_{1} x_{1}^{(i-1)}$ leads to an extended bilinear state space system:

$\dot{x}_{1}=a U_{\text {inc }}$

$\dot{x}_{2}=a U_{\text {azi }} \csc \left(x_{1}\right)=a U_{\text {azi }} \sec \left(\pi / 2-x_{1}\right)=a U_{\text {azi }} \sum_{i=1}^{\infty} b_{i} x_{1}^{(i)}$

where $b_{i}$ are the coefficients of the Taylor series expansion of $\sec (\pi / 2-x)$. The expansion of $\sec (\pi / 2-x)$ is used instead of $\csc (x)$ so that the required powers of $x_{1}^{(i)}$ can be obtained 
from the $\theta_{\text {inc }}$ state equation. The Taylor series expansion of $\sec x$ for $-\frac{\pi}{2}<x<\frac{\pi}{2}$ is obtained from

$$
\sec x=1+\frac{x^{2}}{2}+\frac{5 x^{4}}{24}+\frac{61 x^{6}}{720}+\cdots+\frac{(-1)^{n} E_{2 n} x^{2 n}}{(2 n) !}
$$

where $E_{2 n}$ is the Euler number [13, pp 81-99]. Hence, the Taylor series expansion of $\sec (\pi / 2-x)$ is given as:

$$
\begin{array}{r}
\sec (\pi / 2-x)=1+\frac{1}{2}(\pi / 2-x)^{2}+\frac{5}{24}(\pi / 2-x)^{4} \\
+\frac{61}{720}(\pi / 2-x)^{6}+\cdots+\frac{(-1)^{n} E_{2 n}(\pi / 2-x)^{2 n}}{(2 n) !}
\end{array}
$$

In practice the number of terms in the series expansion and the number of augmented states is truncated to a finite value $N$ (where $N$ is equal for the number of expansion terms and augmented states) to an arbitrary degree of accuracy. Expanding (10), gives the bilinear system:

$$
\begin{aligned}
\dot{x}_{1} & =a U_{\mathrm{inc}} \\
\dot{x}_{1}^{(2)} & =2 x_{1} \dot{x}_{1}=2 a x_{1} U_{\mathrm{inc}} \\
\dot{x}_{1}^{(3)} & =3 x_{1}^{(2)} \dot{x}_{1}=3 a x_{1}^{(2)} U_{\mathrm{inc}} \\
\vdots & \vdots \\
\dot{x}_{1}^{(N)} & =N x_{1}^{(N-1)} \dot{x}_{1}=N a x_{1}^{(N-1)} U_{\mathrm{inc}} \\
\dot{x}_{2} & =a U_{\mathrm{azi}} \sum_{i=1}^{N} b_{i} x_{1}^{(i)}
\end{aligned}
$$

which is in the form of (1); where $A=[], u=\left[U_{\mathrm{inc}}, U_{\mathrm{azi}}\right]^{T}$ and $x=x^{\otimes}$. For example, where $N=3$, the following is obtained:

$$
\begin{aligned}
M_{1}=\left[\begin{array}{cc}
0 & 0 \\
2 a & 0 \\
0 & 0 \\
0 & a b_{1}
\end{array}\right], & M_{2}=\left[\begin{array}{cc}
0 & 0 \\
0 & 0 \\
3 a & 0 \\
0 & a b_{2}
\end{array}\right], \\
M_{3}=\left[\begin{array}{cc}
0 & 0 \\
0 & 0 \\
0 & 0 \\
0 & a b_{3}
\end{array}\right], & B=\left[\begin{array}{ll}
a & 0 \\
0 & 0 \\
0 & 0 \\
0 & 0
\end{array}\right]
\end{aligned}
$$

IV. Control Design

The proposed scheme for the BPI controller design is shown in Figure 2. The BPI controller scheme is inspired by [14], [15] and is a combination of a bilinear compensator and a standard linear Proportional plus Integral (PI) controller. The bilinear compensator is only incorporated on the azimuth feedback loop to account for the nonlinear $1 / \sin \theta_{\text {inc }}$ term in (4).

\section{A. PI Controller}

The PI control for the inclination and azimuth control channels are respectively:

$$
\begin{aligned}
& U_{\mathrm{inc}}=k_{\mathrm{pi}} e_{\mathrm{inc}}+k_{\mathrm{ii}} \int_{0}^{t} e_{\mathrm{inc}} d t \\
& \widetilde{U}_{\mathrm{azi}}=k_{\mathrm{pa}} e_{\mathrm{azi}}+k_{\mathrm{ia}} \int_{0}^{t} e_{\mathrm{azi}} d t
\end{aligned}
$$

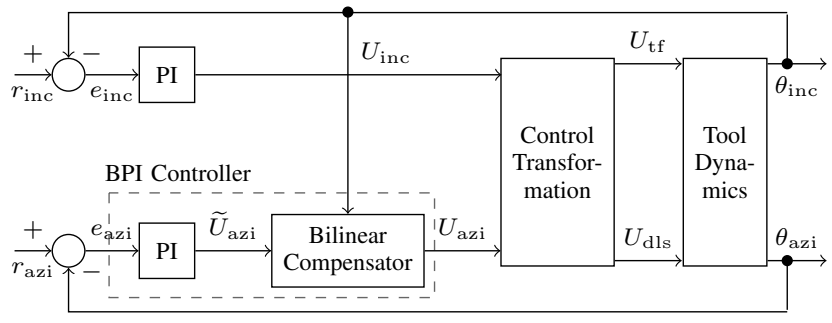

Fig. 2. BPI Control Scheme

where $e_{\mathrm{inc}}=r_{\mathrm{inc}}-\theta_{\mathrm{inc}}$ and $e_{\mathrm{azi}}=r_{\mathrm{azi}}-\theta_{\mathrm{azi}} ; r_{\mathrm{inc}}$ and $r_{\mathrm{azi}}$ are the nominal operating points for inclination and azimuth, respectively.

It is shown in [9] that the gains for the PI controllers in the inclination and azimuth feedback loops can be expressed as:

$$
\begin{aligned}
k_{\mathrm{ii}} & =\frac{\omega_{\mathrm{i}}^{2}}{a}, & k_{\mathrm{pi}} & =\frac{\sqrt{2} \omega_{\mathrm{i}}}{a} \\
k_{\mathrm{ia}} & =\frac{\omega_{\mathrm{a}}^{2}}{a \csc \theta_{\text {inc }}}, & k_{\mathrm{pa}} & =\frac{\sqrt{2} \omega_{\mathrm{a}}}{a \csc \theta_{\mathrm{inc}}}
\end{aligned}
$$

which are dependent on the operating point operating point $a=V_{\mathrm{rop}} K_{\mathrm{dls}}$ and on the chosen natural frequencies of the inclination and azimuth feedback loop dynamics, $\omega_{\mathrm{i}}$ and $\omega_{\mathrm{a}}$ respectively.

\section{B. Bilinear Compensator}

Assuming that $U_{\mathrm{azi}}=\widetilde{U}_{\mathrm{azi}}$, hence, only the PI controller is incorporated (see (16)), the azimuth feedback loop can be expressed based on (10) as:

$$
\dot{\theta}_{\mathrm{azi}}=a \sum_{i=1}^{\infty} b_{i} x_{1}^{(i)}\left(k_{\mathrm{pa}} e_{\mathrm{azi}}+k_{\mathrm{ia}} \int_{0}^{t} e_{\mathrm{azi}} d t\right)
$$

Based on (12) and (13), (19) can further be expressed as:

$$
\dot{\theta}_{\mathrm{azi}}=a\left(1+\frac{1}{2} \beta^{2}+\frac{5}{24} \beta^{4}+\ldots\right)\left(k_{\mathrm{pa}} e_{\mathrm{azi}}+k_{\mathrm{ia}} \int_{0}^{t} e_{\mathrm{azi}} d t\right)
$$

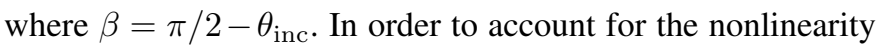
in (20), a bilinear compensator is proposed. The bilinear compensator for the azimuth feedback loop is given as:

$$
\frac{U_{\mathrm{azi}}}{\widetilde{U}_{\mathrm{azi}}}=\frac{1}{1+\frac{1}{2} \beta^{2}+\frac{5}{24} \beta^{4}}
$$

The bilinear compensator, in combination with PI, facilitates the ensuing controller (BPI) to sustain a required degree of control throughout a broader scope of operation about the tuning point compared to that obtained with the PI. Hence, the BPI will give improved invariant azimuth responses compared to that obtained with the PI.

\section{Simulation Results}

In this section, transient responses from simulations using Simulink for the closed loop controlled system using two models are presented. The first model is a 'Low Fidelity Model' and simulation results for a variety of response under different operating conditions and assumptions are presented. 
For comparison purposes, responses of the PI controller without the bilinear compensator are also provided. The second model is a 'High Fidelity Model' which includes the actuator and drill cycle effects briefly discussed in Section II-A. The parameter set used for the simulations are listed in Table I.

TABLE I

Design Parameters And Operating Point Values

\begin{tabular}{ll} 
Parameter & Value \\
\hline$\theta_{\text {inc }}, \theta_{\text {azi }}$ & $\pi / 6 \mathrm{rad}\left(30^{\circ}\right)$ \\
$V_{\text {rop }}$ & $200 \mathrm{ft} / \mathrm{hr}(1.0158 \mathrm{~m} / \mathrm{min})$ \\
$K_{\mathrm{dls}}$ & $8^{\circ} / 100 \mathrm{ft}\left(4.5809 \times 10^{-3} \mathrm{rad} / \mathrm{m}\right)$ \\
$r_{\text {inc }}, r_{\text {azi }}$ & $\pi / 6+0.015 \mathrm{rad}$ \\
$\omega_{\mathrm{a}}$ & $0.0151 \mathrm{rad} / \mathrm{min}$ \\
$\omega_{\mathrm{i}}$ & $0.0121 \mathrm{rad} / \mathrm{min}$ \\
$T_{\mathrm{a}}$ & $0.05 \mathrm{~s}\left(8.333 \times 10^{-4} \mathrm{~min}\right)$ \\
$k_{\mathrm{p}}$ & $1 \mathrm{~s}^{-1}\left(60 \mathrm{~min}^{-1}\right)$ \\
$k_{\mathrm{vp}}$ & 0.5 \\
$k_{\mathrm{vi}}$ & $6 \mathrm{~s}^{-1}\left(360 \mathrm{~min}^{-1}\right)$ \\
$t_{\mathrm{cycle}}$ & $10 \mathrm{~s}(0.1667 \mathrm{~min})$ \\
$\omega_{\mathrm{tf}}$ & $2 \pi \mathrm{rad} / \mathrm{s}(376.991 \mathrm{rad} / \mathrm{min})$ \\
\hline
\end{tabular}

\section{A. Low Fidelity Model Simulation}

Using the nonlinear plant model given by (3) and (4) (with $V_{\mathrm{dr}}$ and $V_{\mathrm{tr}}$ ignored), control architecture and BPI controller design described in Section IV, a Simulink transient simulation was created to show the effectiveness of the proposed BPI controller over the already existing PI controller. In the interest of viewing the dynamic responses, the saturation effect is removed. A reference change of $0.015 \mathrm{rad}$ for a set of various azimuth angles of $\pi / 18 \mathrm{rad}, \pi / 9 \mathrm{rad}, \pi / 6 \mathrm{rad}, \pi / 3 \mathrm{rad}$ and $\pi / 2 \mathrm{rad}$ are implemented to investigate the invariance of the azimuth responses of the BPI and PI. Furthermore, to analyse the effect of time delay on the feedback measurements with respect to the stability and performance on the BPI controller design, the system delays are implemented.

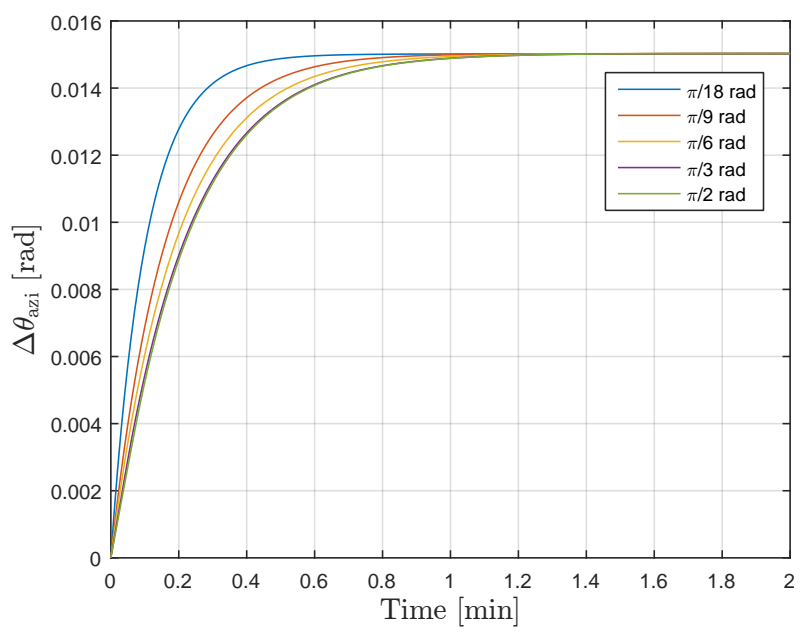

Fig. 3. BPI Azimuth Response

Figures 3, 4, 5 and 6 are responses based on the simulation of the low fidelity model. The BPI and PI azimuth responses for a reference change of $0.015 \mathrm{rad}$ for a set of various azimuth

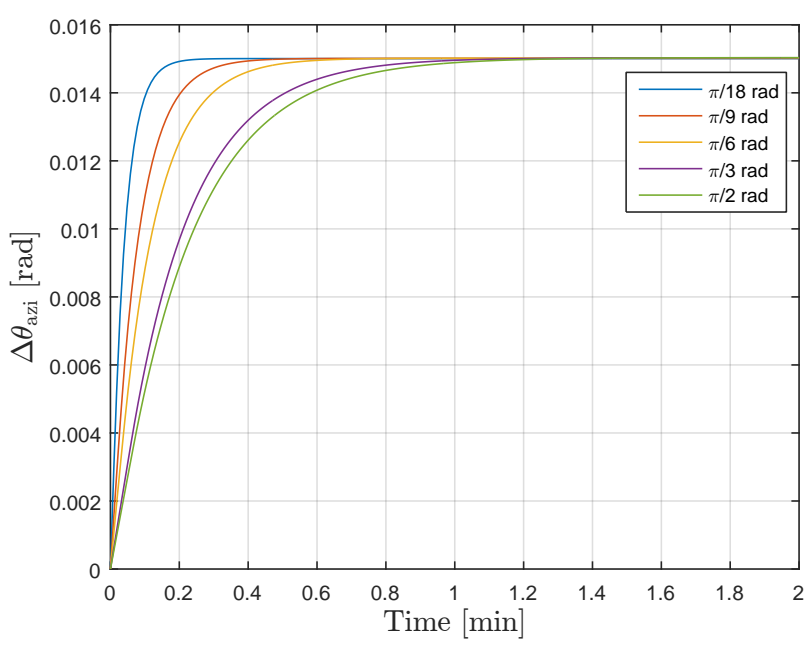

Fig. 4. PI Azimuth Response

angles are shown in Figures 3 and 4, respectively, and where $\Delta \theta_{\text {azi }}(t)=\theta_{\text {azi }}(t)-\theta_{\text {azi }}(0)$. Comparing the azimuth responses of BPI and PI, it can be seen that the azimuth responses for the BPI tend to converge more closely to the nominal operating point of $\pi / 2 \mathrm{rad}$. Hence, the BPI controller gives a more consistent azimuth responses than the PI controller.

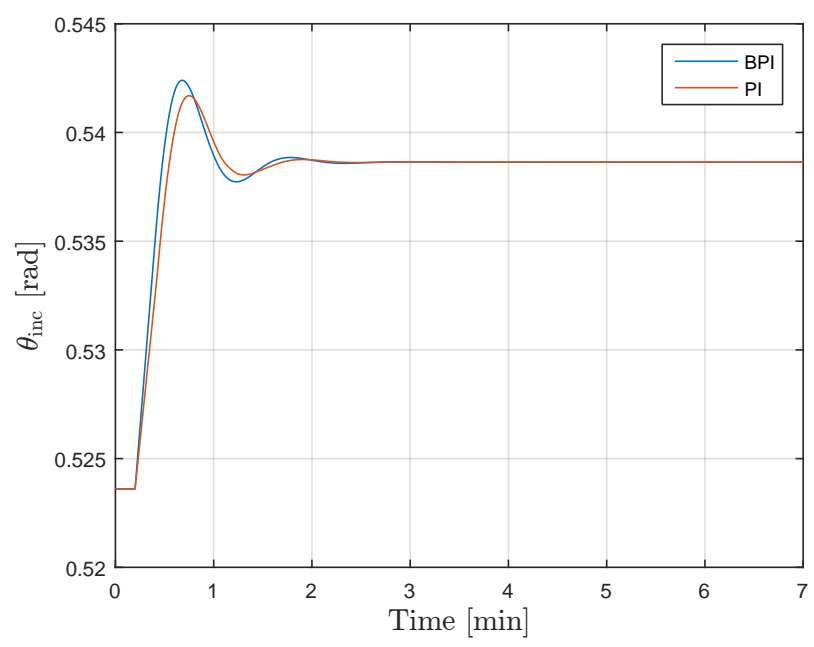

Fig. 5. BPI and PI Inclination Response With Feedback Delay

Figures 5 and 6 respectively show the inclination and azimuth responses with feedback delay of $12 \mathrm{~s}$. As expected, it can be seen in Figure 5 that the inclination responses for both BPI and PI are similar. Nonetheless, in Figure 6, the PI controller exhibits oscillatory characteristics, hence, the azimuth attitude of the directional drilling tool does not converge to the desired angle of $\pi / 6+0.015 \mathrm{rad}$. Conversely, for the BPI controller, the azimuth attitude of the directional drilling tool converges to the desired angle of $\pi / 6+0.015 \mathrm{rad}$. Hence, the proposed BPI controller reduces the adverse effects of time delay on the feedback measurements with respect to 


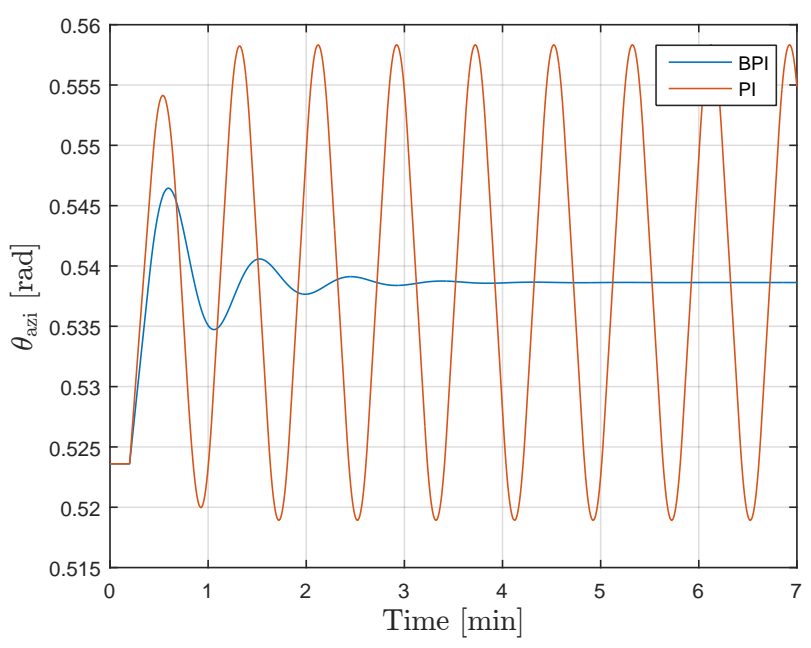

Fig. 6. BPI and PI Azimuth Response With Feedback Delay

stability and performance, as compared to the PI controller (see Figure 6).

\section{B. High Fidelity Model Simulation}

The high fidelity model simulation models the drilling cycle shown in Figure 8 as implemented in [16] and [12], the accelerometers and magnetometers used to derive the azimuth and inclination measurements explicitly and all the system delays and lags previously discussed and detailed in [9]. Based on this, a Simulink transient simulation was created to test directional drilling using the attitude control algorithm to hold azimuth and inclination at the desired angles of $\pi / 6+0.015$ $\mathrm{rad}$ and $\pi / 6+0.015 \mathrm{rad}$, respectively. The high fidelity model architecture is shown in Figure 7.

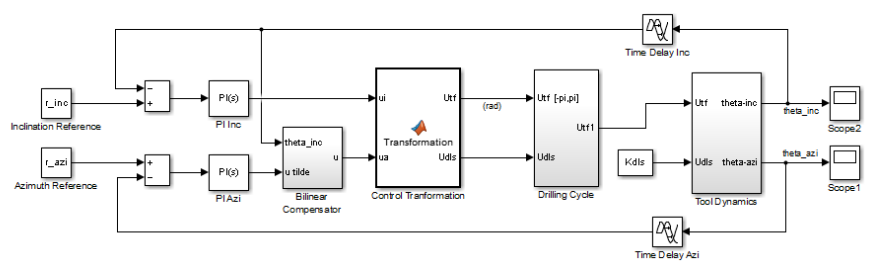

Fig. 7. Simulink Diagram of High Fidelity Model Simulation Scheme

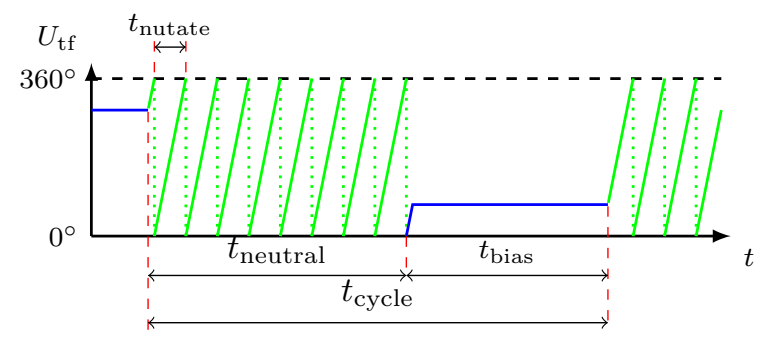

Fig. 8. Drilling Cycle Definition

In order to engineer a variable curvature, $U_{\mathrm{dls}}$, the tool face actuation $U_{\mathrm{tf}}$ (control input) is discretized into duty cycles known as 'drilling cycles' of period $t_{\text {cycle }}$ where in order to approximate the $U_{\mathrm{dls}}$ control input $t_{\text {cycle }}$ is proportioned into the bias, $t_{\text {bias }}$, and neutral $t_{\text {neutral }}$, phases as shown in Figure 8. In the $t_{\text {neutral }}$ phase the $U_{\mathrm{tf}}$ is cycled at a constant rate of period $t_{\text {nutate }}$ whilst in the $t_{\text {bias }}$ phase the $U_{\text {tf }}$ is servo controlled constant. This approximates the $U_{\mathrm{dls}}$ control input as $U_{\text {dls }}=\left(t_{\text {bias }} / t_{\text {cycle }}\right) K_{\text {dls }}$.

The tool-face is driven by a servo mechanism, hence the tool face response to demands in the tool face is subject to lag dynamics. The servo control architecture is as shown in Figure 9, where $T_{\mathrm{a}}$ is the time constant of the servo loop, $k_{\mathrm{p}}$ is the proportional term in servo position loop, and the proportional and integral term in servo velocity loop are $k_{\mathrm{vp}}$ and $k_{\mathrm{vi}}$, respectively.

The servo operates as a position control when in the bias phase and as a speed control in the neutral phase. Integral action is placed in the velocity loop to ensure accurate rotation speed of the tool face in the neutral phase. This means that integral action is not required in the outer loop position control loop, so a proportional controller is used here. This has an added advantage of removing the need for anti windup and bumpless transfer for the switching between position and velocity tracking.

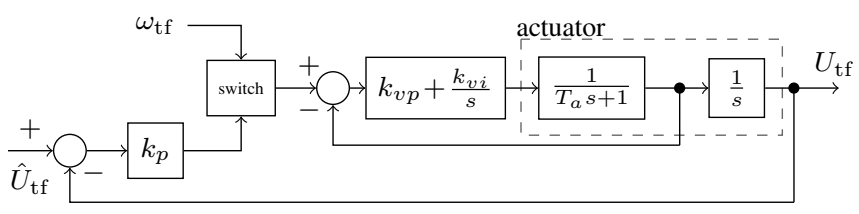

Fig. 9. Toolface Servo Control Architecture

The tool face controller switch is operated so that during the neutral phase, a constant nutate rate demand, $\omega_{\mathrm{tf}}$, is applied to the servo control system speed demand. During the bias phase, the required toolface angle is applied as a reference demand, $\hat{U}_{\text {tf }}$, to the servo control system angle position demand.

Note however, from Figure 10, that at the end of the neutral phase there is a difference between the required toolface angle and the actual toolface angle. Hence, at the start of the drill cycle, there is a change to time, $\Delta t_{\text {neutral }}$ when the switch from $t_{\text {neutral }}$ to $t_{\text {bias }}$ is calculated. From Figure 10, the calculation is straightforward and is given by $\Delta t_{\text {neutral }}=\frac{\Delta U_{\mathrm{tf}}}{\omega_{\mathrm{tf}}}$, where $\Delta U_{\mathrm{tf}} \in[-\pi, \pi)$ is the toolface correction angle given by $\Delta U_{\mathrm{tf}}=\alpha-\operatorname{round}(\alpha, 2 \pi)$, where $\alpha=\omega_{\mathrm{tf}} t_{\text {neutral }}+U_{\mathrm{tf}}(k-1)-U_{\mathrm{tf}}(k-1), U_{\mathrm{tf}}(k)$ represents the toolface demand for the current $\left(k\right.$ th) drill cycle and $U_{\mathrm{tf}}(k-1)$ represents the toolface demand for the previous $((k-1)$ th) drill cycle.

Figures 11 and 12 are the inclination and azimuth responses, respectively, based on the simulation of the high fidelity model with feedback delay of 7.7 s. It can be seen in Figures 11 and 12 that the proposed attitude control algorithm is able to hold the inclination and azimuth of the directional drilling tool at 


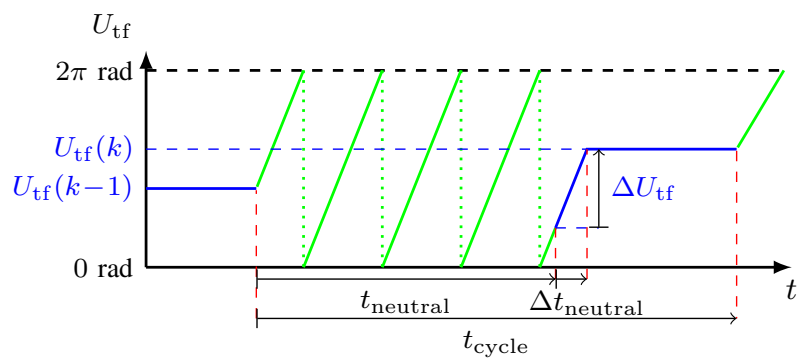

Fig. 10. Calculation of $\Delta t_{\text {neutral }}$ for $k$ th Drilling Cycle

the desired angles of $\pi / 6+0.015 \mathrm{rad}$ and $\pi / 6+0.015 \mathrm{rad}$, respectively (as shown in Table I).

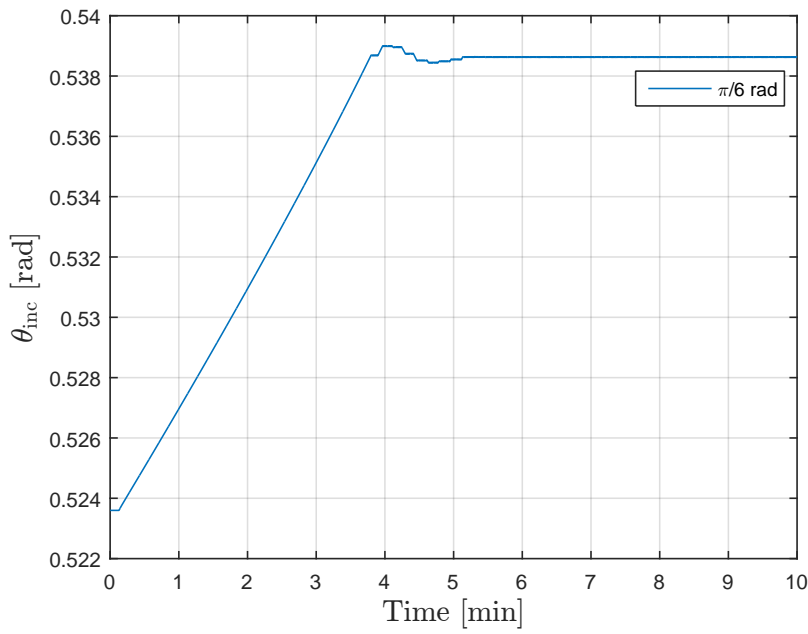

Fig. 11. BPI Inclination Attitude Response

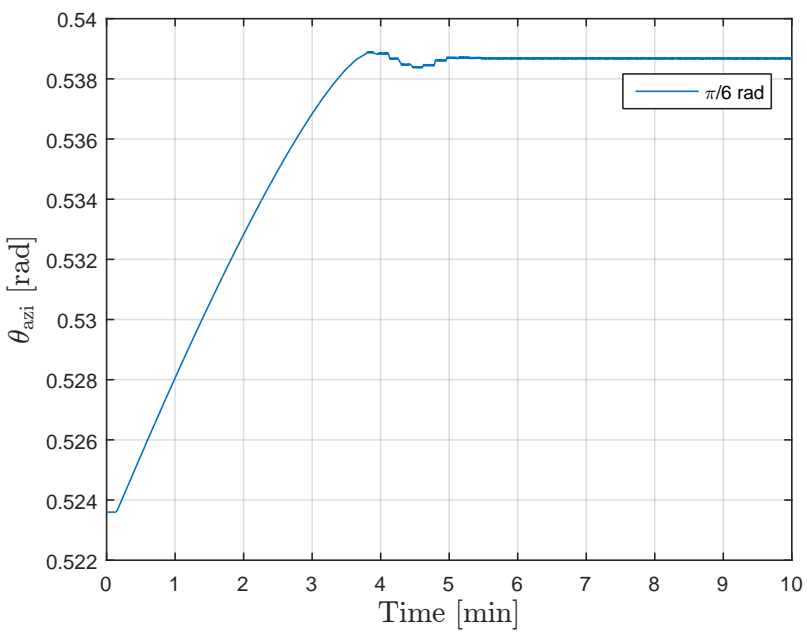

Fig. 12. BPI Azimuth Attitude Response

\section{CONCLUSIONS}

This paper proposes a bilinear model of the directional drilling tool, and also highlights the design of a bilinear controller (BPI) for attitude control of directional drilling tool. This effective implementation shows the possible beneficial aspects of the BPI controller through the improved invariant azimuth responses; and also through the reduction of the adverse effects of time delay on the feedback measurements with respect to stability and performance, as compared to the already existing PI controller. The proposed BPI controller gives more consistent responses over a wider range of operation of the directional drilling tool compared to the already existing PI controller.

\section{ACKNOWLEDGMENT}

To Schlumberger Oilfield UK Plc for funding this research

\section{REFERENCES}

[1] M. Pedersen, M. Lechner, Z. Pon, D. Brink, I. Abbasy, and M. Jaafar, "Successful application of a novel conformance treatment in an extended reach horizontal well in the Al Shaheen field, offshore Qatar," Offshore Europe, September 2009.

[2] T. Yonezawa, E. Cargill, T. Gaynor, J. Hardin, R. Hay, A. Ikeda, and Y. Kiyosawa, "Robotic controlled drilling: A new rotary steerable drilling system for the oil and gas industry," IADC/SPE Drilling Conference, Dallas, Texas, no. 74458, 2002.

[3] U. Shoji, K. Yoshihide, and I. Akio, "Attitude control device and drillingdirection control device," January 1992, patent.

[4] J. Genevois, J. Boulet, C. Simon, and C. Reullon, "Gyrostab project: The missing link azimuth and inclination mastered with new principles for standard rotary BHAs," in SPE/IADC Drilling Conference. Society of Petroleum, Amsterdam, Netherlands, 2003.

[5] L. Yinghui and S. Yinao, "Automatic inclination controller: A new inclination controlling tool for rotary drilling," IADC/SPE Drilling Conference, New Orleans, Louisiana, no. 59259-MS, 2000.

[6] C. Bruni, G. DiPillo, and G. Koch, "Bilinear systems: An appealing class of nearly linear systems in theory and applications." IEEE Transactions on Automatic Control, vol. AC-19, no. 4, pp. 334-348, 1974.

[7] H. Schwarz and H. T. Dorissen, "System identification of bilinear systems via realization theory and its application," Control, Theory and Advanced Technology, vol. 5, no. 2, pp. 137-155, 1989.

[8] M. Ghasemi, A. Ibrahim, and E. Gildin, "Reduced order modeling in reservoir simulation using the bilinear approximation techniques," in SPE Latin American and Caribbean Petroleum Engineering Conference, Maracaibo, Venezuela, 2014, pp. 1069-1078.

[9] N. Panchal, M. T. Bayliss, and J. F. Whidborne, "Robust linear feedback control of attitude for directional drilling tools," in 13th IFAC Symposium on Automation in Mining, Mineral and Metal Processing, 2010.

[10] B. Kim and M. Lim, "Robust $\mathrm{H}_{\infty}$ control method for bilinear systems," Int. J. of Control, Automation and Systems, vol. 1, no. 2, pp. 171-177, 2003.

[11] M. T. Bayliss, J. F. Whidborne, and N. Panchal, "Structured uncertainty analysis of pole placement and $H_{\infty}$ controllers for directional drilling attitude tracking," IFAC World Congress, vol. 19, no. 1, pp. 9283-9288, 2014.

[12] M. T. Bayliss, I. J. Inyang, and J. F. Whidborne, "Application of LQG control to attitude control of directional drilling," in The 24th International Conference on Systems Engineering, Coventry, U.K., 2015.

[13] L. Rade and B. Westergren, Mathematics Handbook for Science and Engineering, 4th ed. Berlin - Tokyo: Springer, 1999.

[14] S. Martineau, K. J. Burnham, J. A. Minihan, S. Marcroft, G. Andrews, and A. Heeley, "Application of a bilinear PID compensator to an industrial furnace," in 15th IFAC World Congress on Automatic Control, Barcelona, Spain, 2002.

[15] S. Martineau, K. J. Burnham, O. C. L. Haas, G. Andrews, and A. Heeley, "Four-term bilinear PID controller applied to an industrial furnace," Control Engineering Practice, vol. 12, no. 4, pp. 457-464, 2004.

[16] M. T. Bayliss and J. F. Whidborne, "Mixed uncertainty analysis of pole placement and $\mathrm{H}_{\infty}$ controllers for directional drilling attitude tracking," Journal of Dynamic Systems, Measurement, and Control, vol. 137, no. 12, p. 121008, 2015. 
2016-11-10

\section{Bilinear modelling and bilinear PI control of directional drilling}

Inyang, Isonguyo J.

IEEE

Inyang, I.J., Whidborne, J.F., Bayliss, M.T. (2016) Bilinear modelling and bilinear PI control of directional drilling. 2016 UKACC International Conference on Control, UKACC Control 2016, 31

August 2016 - 2 September 2016, Article number 7737607

https://doi.org/10.1109/CONTROL.2016.7737607

Downloaded from Cranfield Library Services E-Repository 